\title{
Focalización y movilidad social: una revisión del caso colombiano
}

Luis Eduardo Reina-Bermúdez

Universidad Nacional Abierta y A Distancia 


\title{
Focalización y movilidad social: una revisión del caso colombiano
}

\author{
Luis Eduardo Reina-Bermúdez \\ Universidad Nacional Abierta y A Distancia - Colombia
}

Para citaciones: Reina Bermúdez, L (2021). Focalización y movilidad social: una revisión del caso colombiano. Panorama Económico, 29(2), 145-159.

Recibido: 1 de febrero de 2021

Aprobado: 30 de marzo de 2021

Autor de correspondencia:

Luis Eduardo Reina-Bermúdez

lerbermudez@gmail.com

Editor: Andrés Escobar E. Universidad de Cartagena-Colombia.

\section{Tipología IBN Publindex:}

Artículo de Reflexión

Copyright: (C 2021. Reina Bermúdez, L. Este es un artículo de acceso abierto, distribuido bajo los términos de la licencia https://creativecommons.org/licenses/bync-sa/4.0/ la cual permite el uso sin restricciones, distribución y reproducción en cualquier medio, siempre y cuando que el original, el autor y la fuente sean acreditados.

\section{RESUMEN}

Este articulo analiza los procesos de focalización en las políticas públicas y cómo estos, permiten generar movilidad social. Se retoma la discusión ética sobre las implicaciones de la validez de la focalización versus la universalización, examinando los costos sociales asociados. Con ello, se plantea brevemente la necesidad de focalizaciones territoriales. Finalmente, se discute sobre un mecanismo de focalización estandarizado en Colombia: el SISBEN IV; versión a la cual se ha llegado no solo por la normativa de actualización periódica, sino también por la necesidad de corregir prácticas de corrupción que llevaron a beneficiar a población que no debería ser objeto de programas sociales.

Palabras clave: Focalización; movilidad social; políticas públicas.

\section{Targeting and social mobility: a review of the colombian case}

\section{ABSTRACT}

This article analyzes the processes of targeting in public policies and their effects on social mobility. The ethical implications of the validity of targeting versus universalization are described, discussing the associated social costs. With this, the need for territorial regionalization is briefly raised. Finally, a standardized targeting mechanism in Colombia is discussed: SISBEN IV, a method that has been reached not only because of the regulations that are periodically updated, but also because of the need to correct corruption practices that led to inadequate targeting of beneficiaries of social programs.

Keywords: Targeting; social mobility; public policies. 


\section{INTRODUCCIÓN}

Dados los recursos escasos, la acción de política pública que adelantan los gobiernos requiere concentrarse en la atención de grupos poblacionales determinados, apelando tanto a la utilidad marginal del dinero benthamiana (Cummins, 2019) como a la racionalidad económica. Adicionalmente, desde el siglo XX el Estado y sus dependencias -incluyendo entidades territoriales - enfrentan crecientes demandas por transparencia y democratización de derechos, presionando actuaciones guiadas por la racionalidad económica, lo que ha dado relevancia a los procesos de focalización. Estos procesos de focalización generan movilidad social, asunto que se constituye en la hipótesis de este artículo.

Para mostrar esto, el articulo presenta el concepto de focalización, acompañado de algunos ejemplos en el contexto latinoamericano. Se discute, además, la ética de los procesos de focalización, y se indica que también debería tener criterios territoriales. Posteriormente, se explica el sistema de registro que permite realizar focalización del gasto social en Colombia: el SISBEN. Se cuestiona si este contempla un actuar en los diferentes frentes de la movilidad social —el tributario, salud, alimentación, educación, así como en materia de trabajo y mejoramiento de ingresos. Ello permite mostrar cómo se han ampliado programas sociales con criterios de focalización. Sin embargo, a la luz del sobrerregistro de personas en los datos disponibles, se puede deducir una ineficiencia en ese gasto público. Con lo anterior, se allana el camino para una respuesta empírica a la pregunta sobre si el gasto social genera movilidad social en nuestro país.

\section{LA FOCALIZACIÓN ES...}

La focalización, o targeting en inglés, de acuerdo con Thomas Nechyba (2000), consiste en la conversión de un determinado grupo social en destinatario de cierta política pública, de acuerdo con criterios previamente establecidos, entre los que se suele incluir el sufrir de privaciones que implican pobreza. Otra visión es que se trata de grupos marginados, es decir, excluidos o en riesgo (Torres-Melo y Santander, 2013). Esta forma de implementar programas sociales constituye una alternativa a las políticas universalistas. Alternativa que gana fuerza como consecuencia de la crisis de los años 1980s, particularmente en américa latina se le ha denominado la década perdida. Por recomendación de organismos multilaterales, como el Banco Mundial, en lugar de esparcir el gasto social en el conjunto de la población se concentra en los más vulnerables (Salgado, Martínez y Meireles, 2018).

Ahora bien, no necesariamente se focaliza o direcciona el accionar público por criterios de pobreza. En algunos casos se establecen acciones enfocadas en ciertos sectores económicos en apuros, o para los que el apoyo resulte estratégico. En dado caso, se trata de políticas sectoriales, como las de la cadena láctea o del caucho. Sin embargo, se tienden a implementar políticas públicas que buscan beneficiar únicamente a ciudadanos y familias que viven abajo de cierto umbral de pobreza y, por tanto, están en riesgo social (Raczynski, 1995; Subramanian, 2019). Como indica Fernando Filgueira (2014), "las políticas focalizadas poseen el mérito de concentrar los recursos escasos en las poblaciones que realmente lo requieren" (p. 16); con lo que se consigue mejorar el bienestar social (Reina, 2017a). 
Por cuenta de lo anteriormente expuesto, se considera que la focalización busca mejorar algún fenómeno social que tiene un efecto negativo en una comunidad o grupo humano. Dicha mejora ocurre al encaminar acciones que impactan directa e indirectamente determinados indicadores sociales asociados al fenómeno social, cuyos niveles actuales pueden señalar la presencia de un riesgo social (González, 2000; Reina, 2017b). Cuando la focalización se hace con datos precisos en reconocimiento de la complejidad de la situación de riesgo, las acciones estatales tendrán mayor impacto, medido en términos de eficiencia y efectividad en redistribución de ingresos y disminución de la pobreza. Esto ocurre, porque, siguiendo el utilitarismo de Jeremy Bentham, una determinada cantidad de riqueza trasladada a los más pobres generará incrementos de bienestar proporcionalmente mayores que los potencialmente producidos en capas sociales de ingresos superiores (Rivera, 2011; Filgueira, 2014).

Lo anterior parece relativamente sencillo, pero es importante reconocer que la focalización tiene desventajas y costos por tener en cuenta en el momento de crear y continuar políticas sociales. Estos costos y desventajas son diversos, y entre ellos se pueden contar la posible manipulación de información, las distorsiones en incentivos que provoquen efectos adversos, des-utilidades, estigmas, pérdidas administrativas, la exposición de la vida privada e, incluso, problemas de gobernabilidad (Hernández, Orozco y Vázquez, 2005; Torres-Melo y Santander, 2013). Estos factores negativos pueden reforzarse unos a otros, restringiendo o anulando las intenciones de la focalización (Sen, A., 2003). Ocurre, además, que algunos grupos sociales vulnerables pueden no tener la capacidad de hacerse incluir en la agenda pública o para los que, desde el Estado —en el caso en que se logre generar para ellos una política social—, sea difícil mantener el apoyo de una focalización que los beneficie, ${ }^{1}$ lo cual puede afectar la cohesión social (Huber y Stephens, 2004). Esto entraña un costo político que los gobiernos no siempre están dispuestos a asumir.

La calidad de información es otro factor de costos por tener en cuenta. La baja calidad o inexistencia de información objetivo podría llevar a prácticas de auto-reporte y a la identificación errónea de algunas unas personas como beneficiarios. Las versiones anteriores de SISBEN son un ejemplo de fallas en la metodología de auto-reporte, pues personas con capacidad de pago de su seguridad social terminaban con beneficios en materia de salud subsidiada. Los incentivos adversos constituyen otro costo social, el cual llevaría a algunas personas a mantenerse entre los beneficiarios, incluso cuando ello implique mantener su pobreza. Por ejemplo, incentivos dados por número de hijos podrían desestimular el control de la natalidad con las consecuencias que ello tiene sobre las posibilidades de calidad de vida que las familias le pueden brindar a sus hijos.

Entonces, es necesario construir información fiable, con mecanismos de respuesta amplia que permitan identificar acertadamente a los beneficiarios. No hacerlo entraña un costo administrativo y ético: destinar recursos a quien no lo requiere (Hernández et al., 2005). Aunque no existe una focalización perfecta, siempre se debe buscar la alternativa más eficiente y justa, que maximice el beneficio de los más vulnerables. Para ello, además de tener en cuenta los costos listados en los párrafos anteriores, se deben incluir también

${ }^{1}$ Sobre el respecto se quiere resaltar que toda política económica es una política social, entendida esta en un sentido amplio como bien señaló Jorge Iván González (2000). Se puede revisar, además, lo expuesto por Torres-Melo y Santander (2013). 
elementos tradicionales en las políticas públicas, tales como los incentivos, la viabilidad tecnopolítica del programa y su efectividad en sacar de la pobreza a los beneficiarios.

Por ello, en la focalización suele ser necesario usar herramientas técnicas, estadísticas y econométricas. Lo cual, realizado adecuadamente, constituye la modernización estatal. A través de la correcta gestión de información se evitará la atomización del gasto público y la duplicidad de programas sociales. Para finalizar esta sección, debe señalarse que los problemas o dificultades para implementar mecanismos de focalización y promover la movilidad social se hacen más grandes entre menores niveles de desarrollo económico tenga un país. Es decir, un menor tamaño de la economía de los países y una mayor incidencia de la pobreza dificultan la focalización. También incide el peso de la economía informal, pues los trabajadores en dicho sector están mayormente expuestos a los efectos negativos de contingencias de salud y de crisis económicas.

En últimas, focalizar es una necesidad ante la escasez de recursos (Stiglitz, 2003). Aunque existe evidencia que indica que allí donde hay más programas sociales focalizados hay más pobreza y menor esfuerzo fiscal (Huber, 2002).

\section{FOCALIZACION EN EL CONTEXTO LATINOAMERICANO}

Existen diferentes programas focalizados hacia los más vulnerables. Muchos de ellos implican entrega o acceso a bienes y servicios. Los programas de ampliación de cobertura de educación y salud son un ejemplo de ellos (De Hoyos, Attanasio y Meghir, 2019). Otro ejemplo es el suministro de alimentos y utensilios para dormir o para el aseo en situaciones de calamidad por desastres naturales, de guerra o de migración externa o interna. Esto ayuda a compensar su demanda y mantener niveles mínimos de bienestar, cuando ello no pueda ser conseguido por vía del trabajo y del mercado (Filgueira,2014; Aguado, 2015). Particularmente en América Latina, los conflictos internos y la situación económica de sus países han generado olas de población desplazada que requieren de estas ayudas inmediatas. Lastimosamente, esta dinámica de migración genera un desbalance entre oferta y demanda de trabajo, solventada a medias con emprendimientos informales. Adicionalmente, crece la mancha urbana y la necesidad de diferentes políticas de ampliación de servicios públicos y políticas sociales (Torres-Melo y Santander, 2013).

También existen los internacionalmente denominados programas de transferencias condicionadas de recursos — TCR en español, o CCT por las siglas en inglés para Conditional Cash Transfers - Muchas de las TCR son orientadas a personas adultas mayores o niños, niñas y adolescentes (Asri, 2019). Bolsa Familia (Brasil), Prospera (México) y Familias en Acción (Colombia) son programas de TCR que se han llevado a cabo en Latinoamérica (Salgado, Martínez y Meireles, 2018). En general lo que ha sucedido es que se genera un registro estandarizado que permite articular y coordinar, por supuesto direccionar la oferta de programas sociales a población en condiciones de pobreza. Aunque existen variados esquemas de corresponsabilidad, lo importante es que se está prestando atención a ello y que estos programas están permitiendo el goce de los derechos humanos. Para cerrar ese panorama latinoamericano se debe señalar los diferentes programas y proyectos de diferentes ONG. Dichas acciones focalizadas, sin embargo, no consiguen homogenizar disminuir las enormes desigualdades en estos 
países (Senhoras, 2003). En la misma vía excesos de focalización por las leyes federales en el caso de Brasil conllevan a la pulverización del gasto (ibid).

\section{LA ÉTICA DE LA FOCALIZACIÓN}

Las políticas públicas, especialmente las sociales, y su correcto funcionamiento legitiman al Estado (Torres-Melo y Santander, 2013). Ellas construyen la realidad social y son esenciales para conseguir el desarrollo (Roberts, 2001). Por ello, se insiste en la focalización y su seguimiento, en especial en países que constitucionalmente se han comprometido a ser garantistas de derechos humanos, como es el caso de Colombia y de muchas otras repúblicas que han modificado sus cartas políticas desde la profundización de la globalización en los años noventa. En estos países, se estableció el reconocimiento de los derechos de cuarta generación, también denominados económicos, sociales y ambientales —DESCA— (Torres-Melo y Santander, 2013). Dichos cambios promueven la expansión de las libertades afines al desarrollo con un sentido humano (Sen, 1999; Max-Neef, Elizalde y Hopenhayn, 2006). En esa medida, se podría argumentar que, debido al avance en protección de derechos humanos, no se deberían focalizar ciertas acciones de política pública, sino que lo procedente es la universalización (Fonseca, 2008; Devereux, 2016). Asunto que se resuelve con el llamado a la progresividad en la garantía de los derechos, de acuerdo con las capacidades presupuestales de los Estados (Pautassi, 2020; Ferrer, 2020)².

Esta conciliación fue necesaria e incorporada en las constituciones latinoamericanas de los años 1990s por las fuertes restricciones fiscales que dichos países tuvieron en la década de 1980. Principalmente fueron una solución por los cada vez mayores sectores públicos, burocratizados, demandando crecientes recursos. Esto además porque la seguridad social siempre había estado per sé a los sectores formales y de facto no se tenía universalización para la población en los sectores informales de la economía (Ocampo, 2008). En este marco de progresividad y racionalidad económica, el ejercicio adecuado de la focalización podría permitir el direccionamiento de los esfuerzos estatales de forma transparente (Hernández et al., 2005). Esto es especialmente cierto cuando se crean criterios claros y objetivos para incorporar o descartar a personas u organizaciones en el grupo beneficiario de la política social. En muchas ocasiones, cuando las políticas públicas se realizan sin focalización, los beneficios pueden no llegar a aquellas personas que más requieren ayuda: los más pobres entre los pobres.

Otro aspecto ético que atraviesan los procesos de focalización son los problemas de acceso y asimetrías en la información a los que se enfrentan las personas más pobres; situación que les impide conocer cuáles son los requisitos que deben cumplir para beneficiarse de programas sociales. Esto es resultado de bajas capacidades socioculturales, falta de habilidades para manejo de tecnologías e, incluso, del diseño burocrático del proceso de selección de beneficiarios. Por tanto, una focalización ética debe tener en cuenta las capacidades socioculturales y tecnológicas de la población beneficiaria, las cuales posibilitan o inhiben el acceso a información de los programas sociales.

\footnotetext{
${ }^{2}$ Ver Sánchez Cubides (2018). Recordar que los principios de los derechos humanos son: universalidad, igualdad y no
} discriminación, exigibilidad, indivisibilidad, interdependencia, inalienabilidad, progresividad, diversidad y participación. 
Infortunadamente, como lo señalan Hernández et al. (2005) ocurre que las políticas públicas

han tendido a satisfacer las demandas de los grupos que tienen menores necesidades, pero cuya ubicación territorial tiene más fáciles accesos, cuentan con organización social y política, o disponen de mayor información para acceder a los programas y proyectos sociales. (p.5)

Esto pone de presente que una buena política pública debería tener en cuenta aspectos territoriales en la focalización. Se requiere de un análisis que involucre no solo variables estándar, sino también aquellas que evidencien desigualdades entre los territorios de cada país, con el fin de avanzar en materia de solidaridad interterritorial (Franco y Reina, 2018). Por ello, se considera que la focalización se inserta en una discusión ética mayor que la asociada con el eterno debate entre equidad y eficiencia (Stiglitz, 2003). En todo caso, el gobierno puede elegir un criterio único, asignar a alguno mayor peso o tomar varios de ellos en consideración para decidir la inclusión de beneficiarios.

\section{LA FOCALIZACIÓN DEBERÍA TENER CRITERIOS TERRITORIALES}

La ubicación en ciertas zonas -especialmente urbanas y capitales nacionales o de departamentos - permite a las personas un acceso relativamente fácil a oficinas estatales y a información. Por su parte, en otros lugares, por distancias o costumbres, de facto existe una restricción en términos de información, que no siempre se tiene en cuenta en los diferentes programas sociales. Esto sucede porque los planificadores se interesan en abultar sus indicadores de productos y resultados, buscando un aparente criterio de eficiencia. Resulta más fácil mostrar mayores resultados, si se atiende población que está concentrada, aunque se margine una vez más a quienes viven en las periferias espaciales.

La focalización podrá tener criterios territoriales en función de la calidad de la información, como aquella que explica cómo se distribuye espacialmente la pobreza (Cortés, 2014). Dicha disponibilidad de información contribuirá a estudiar la convergencia nacional de la pobreza e, incluso, motivaría la focalización a escala micro territorial (Ruiz, Botello y Marín, 2014). El número de organizaciones sociales, graduados universitarios, homicidios y desplazamientos están relacionados con el crecimiento económico (LlanesDueñas, 2017). Las dos primeras variables se correlacionan positivamente mientras que las segundas se correlacionan negativamente. Estas variables son eminentemente territoriales y de hecho ya son objeto de focalizaciones de acciones de los cuerpos de seguridad. En este sentido decir que la focalización en programas sociales debe ser territorial constituye solo una ampliación de dichas acciones de seguridad.

También se sabe hoy día que el pertenecer a familias grandes, de actividad agropecuaria, así como pertenecer a una minoría étnica y vivir en una región pobre se correlacionan negativamente con los niveles de ingreso individuales (Herrera-Jiménez, A., \& VillegasQuino, H. (2016). Las comunidades étnicas normalmente tienen una localización especifica por lo que focalizar con criterios territoriales, de nuevo, cobra sentido. De alguna manera, esto ya se realiza en Colombia a través de las trasferencias del Sistema General de Participaciones y del Sistema General de Regalías. Sistemas que incluyen criterios de pobreza y tamaño poblacional para calcular las transferencias del gobierno central hacia las entidades territoriales, lo que constituyen parte de lo que Irma Franco y 
Luis Reina (2018) denominan la emergencia de la solidaridad interterritorial en Colombia. La idea no es nueva en realidad, encontramos en Brasil y las cotas de acceso a la educación superior estatal una forma de focalización, guiada por la discriminación positiva. El sistema consiste en que cada estado de la federación adecua sus cuotas de acceso acorde a su composición racial (Senhoras, 2015).

\section{¿CÓMO SE FOCALIZA EN COLOMBIA?: EL CASO DEL SISBEN IV}

El Sistema de Identificación de Potenciales Beneficiarios de Programas Sociales SISBEN- es, por mandato de ley en Colombia, el mecanismo más utilizado para focalizar el accionar de las políticas sociales. Este sistema es diseñado por la nación, a través del Departamento Nacional de Planeación (DNP). Cada tres años, el DNP debería actualizar y mejorar la metodología a través del uso de herramientas estadísticas que faciliten la identificación y ordenación de la población, por sus condiciones socioeconómicas, tras haber respondido la encuesta del SISBEN. Se reconoce esto en virtud de lo establecido en el artículo 24 de la Ley 1176 de 2007, que reza: "El Conpes Social definirá cada tres años los criterios e instrumentos para la determinación, identificación y selección de beneficiarios, así como, los criterios para la aplicación del gasto social por parte de las entidades territoriales". En consecuencia, el DNP, como órgano técnico de apoyo al Consejo Nacional de Planeación Económico y Social (CONPES), ha venido actualizando la metodología.

El problema del SISBEN, sin embargo, es su delegación en las alcaldías y la aparición de fenómenos de corrupción. Algunos ciudadanos, por ejemplo, dan falsos reportes de sus condiciones socioeconómicas para obtener puntajes convenientes dentro del sistema. Por su parte, se ha visto que algunos funcionarios incluyen a simpatizantes políticos en el SISBEN. Pero existe, además, un problema técnico, pues las alcaldías no siempre tienen la capacidad para registrar y levantar la información de forma adecuada. Esta falla abre campo a la corrupción, materializada principalmente en el registro de personas que no cumplen con los criterios esenciales para ser beneficiarios de políticas sociales, lo que se conoce como sobrerregistro.

En particular, los problemas de las metodologías anteriores a SISBEN IV se asociaban con la falta de cruces de información con otras fuentes, lo que producía una selección incorrecta de beneficiarios de los programas sociales. De hecho, en el Plan Nacional de Desarrollo 2018-2022 se señala que, antes de la depuración de SISBEN IV, existían 36 millones de personas inscritas, cifra que resulta cuestionable al considerar, por ejemplo, que el estimado de población bajo la línea de pobreza monetaria en el país es de 13 millones (Clavijo, Vera, Cuéllar y Joya, 2019). El actual sistema, SISBEN IV, cuya actualización ocurrió en $2016^{3}$, toma en cuenta tanto lo productivo como lo social, con el fin de facilitar la identificación de personas pobres y vulnerables en todos los municipios colombianos. Aunque no es la intención explicar aquí la metodología empleada para actualizar el registro, sí se considera pertinente indicar que este se renovó, al menos en Bogotá, a partir de un censo en barrios de estratos 1, 2 y 3. Como este, se realizaron ejercicios similares en otros municipios del país. La innovación central es que la

\footnotetext{
3 Para ahondar sobre la metodología, se puede revisar el leer el Documento CONPES 3877 de 2016. Adicionalmente, puede consultarse la Ley 715 de 2001, especialmente el artículo 94.
} 
información suministrada por las personas encuestadas para ingresar en el sistema se cruza con otras bases de datos para descartar a los no vulnerables.

Entre 2017 y 2019 se tuvo en el país un período de transición hacia la actualización de los registros, permitiendo, al menos en teoría, la depuración y la superación de la ineficiencia en el gasto social al garantizar que las ayudas llegaran a la población que realmente lo necesitaba. Por tanto, sería de esperar que la última versión, además de confiable y efectiva deba estar ya actualizada y validada por la metodología oficial. Por lo anterior, actualmente, los municipios deberían usar un registro con base en la cuarta versión metodológica del SISBEN, con la que se espera la garantía de llegar a población en condiciones de pobreza. Por ejemplo, en el contexto de la pandemia por el COVID-19 varios municipios acudieron a esa base de datos.

Sin embargo, existe un problema en la celeridad de las actualizaciones de registro, ya que su implementación depende de los presupuestos municipales. Así, en la mayoría de los casos, los municipios terminan por realizar levantamientos incompletos de información. En la práctica, esto supone que muchos municipios continúan basándose en los reportes que dan los mismos ciudadanos (sin cruzar la información) o en los datos recopilados en la tercera versión del sistema de registro. Lo anterior denota la persistencia de un tema político en el trasfondo. Al dejarse la responsabilidad del registro a las alcaldías no se garantiza una ejecución totalmente técnica, como sucedería si se encargara de ello el Departamento Administrativo Nacional de Estadística —DANE- o el DNP. Lastimosamente, el delegar esta tarea a instituciones municipales, y no nacionales, hace que persista el riesgo moral. Se considera que subsiste un incentivo para ayudar a sisbenizar ${ }^{4}$ a población favorable a los propósitos políticos del partido de gobierno, dando cabida a la continuidad del sobrerregistro sin atender a los criterios objetivos para focalizar el gasto social.

Adicionalmente, causa curiosidad que se haya delegado la responsabilidad de actualización de los datos a los mismos beneficiarios registrados en el SISBEN. Con lo anterior, se espera que, si mejora la situación económica de una persona registrada, sea ella misma quien lo reporte a la alcaldía municipal. Aquí, de nuevo, se configuran un riesgo moral, esta vez del lado de los beneficiarios. Para cerrar esta sección se indica que, en Colombia, con base en el registro del SISBEN se han implementado programas sociales como la afiliación al sistema de salud subsidiada y transferencias monetarias condicionadas (Garzón, 2018; Zapata y Valencia, 2018; Ibarra, 2019). Es decir, han existido programas sociales basados en focalización, al menos en este siglo XXI, que se convierten en alternativas para el desarrollo frente a los fracasados modelos de Industrialización por Sustitución de Importaciones - $|S|$ - y los subsidios a la oferta de diferentes bienes meritorios (Reina, 2018). Sería necesario mirar si ellos han generado movilidad social.

\section{¿LA FOCALIZACIÓN DE GASTO SOCIAL EN COLOMBIA GENERA MOVILIDAD SOCIAL?}

La movilidad social se define como el movimiento de un individuo, o su familia, entre las clases o estratos de un espacio social ordinal. Cuando dicho movimiento acontece a lo largo de la vida de un individuo se denomina intrageneracional, y cuando ocurre con su

${ }^{4}$ Verbo de uso común en Colombia para denotar la inclusión de personas o grupos poblacionales dentro del SISBEN con los puntajes necesarios para ser beneficiarios de políticas públicas. 
progenie (hijos, normalmente) se considera intergeneracional. A nivel internacional, las variables de proxy social para medir la movilidad intra e intergeneracional han sido: la ocupación, el nivel educativo y de ingresos. La movilidad social es función de los sistemas educativo y tributario, de políticas sociales y de la inclusión financiera. Se espera, así, la existencia de una educación pública, financiada parcial o totalmente por el Estado, y que favorezca la formación técnica y profesional. Dinamiza a la movilidad social, igualmente, un sistema tributación progresivo, así como la creación de escenarios en los que los grupos sociales de menos ingresos cuenten, al menos, con acceso a crédito para cualificarse o establecer negocios. También se puede apoyar la mejora socioeconómica de los individuos con la implementación de políticas, programas y proyectos sociales, teniendo en cuenta criterios de equidad.

Además, la movilidad social está íntimamente ligada con los fenómenos sociales de desigualdad y de pobreza, los cuales deben ser objeto de atención por parte de investigadores y planificadores del desarrollo. La desigualdad extrema haría más difícil grandes saltos entre los grupos sociales, medidos por quintiles o deciles. En este articulo se elude la discusión de movilidad entre quintiles o deciles de población, por considerar que resulta problemático para medir impactos de políticas públicas y, en últimas, de los progresos o retrocesos en el cambio social. Por el contrario, se deben establecer puntos objetivos fijos para verificar si los estándares de vida mejoran o empeoran. Lo anterior llevaría a revisar, por ejemplo, si las poblaciones en condiciones de pobreza o con ingresos medios aumentan o disminuyen, tanto en términos porcentuales como absolutos. A pesar de la pérdida de correspondencia directa con los asuntos de la movilidad social, se podría llegar a responder la pregunta planteada en este apartado, ya sea mediante argumentación —como se realiza en el presente articulo- o con una estrategia econométrica.

Se debe señalar que el gasto social ha sido insuficiente $y$, muchas veces, errado (Alzate, 2011). Desde luego, esto tiene que ver con la inclusión errónea de beneficiarios en los registros de las versiones 1, 2 y 3 del SISBEN. Esto conllevó, hasta ese momento, a la inviabilidad de una focalización técnica que pudiera substraer efectivamente a las personas de la pobreza. Aunque en este artículo se omite algún ejercicio de estadística correlacional, en virtud de lo objetivos ilustrativos y teóricos trazados desde el inicio, la realidad es que ha sido muy baja la movilidad social en el país. De hecho, en el período 1980 -2010 el 20\% de la población con mayores ingresos del país solo disminuyo en 2\% su participación en el ingreso pasando del 62,71\% a 60.15\% (Cuadro-Guzmán \& JiménezMartínez, 2016, p. 154). Tal es la conclusión a la que llega Ayala (2017) tras una revisión de estudios de medición, quien asegura que:

En Colombia, la medición de la movilidad social ha sido estudiada considerando como clase social el nivel educativo (Angulo et at., 2014; Bonilla, 2011; Gaviria, 2002; Cartagena, 2005; Viafara et al. 2010), la ocupación (Urrutia y Gómez, 1981; Santa María et al., 2009) algunas medidas del status socioeconómico (Galvis y Meisel, 2014) y estratos basados en percepción (Londoño, 2011). Todos los autores coinciden en que la movilidad social en Colombia es baja, más aún si se compara con lo que se observa en otros países latinoamericanos. (p. 109)

Sin embargo, es importante reconocer que, al menos en la segunda mitad del siglo XX, la sociedad ha tenido algunos cambios sociales positivos, como el aumento de la 
expectativa de vida y de niveles de alfabetización, acercándose a lo observable en países desarrollados (Gaviria, 2010). Desde luego, el problema del progreso social se articula, en el caso colombiano, con el conflicto interno, alimentado por el narcotráfico, pero también, como relata Édgar Revéiz (2016), por la cooptación sucesiva del Estado. Alimentada por el conflicto armado, se presenta la desigualdad, que coincide con las pocas oportunidades de ascenso social (Angulo, Azevedo, Gaviria y Páez, 2014). Esta situación se agrava, si se considera que las oportunidades para ascender socialmente, además de ser en conjunto bajas para los grupos de ingresos inferiores, son incluso menores para la población con colores de piel oscuras (Viáfara, 2017).

Pese a esto, considerando puntos fijos para establecer límites de clase - sin seccionar la población por quintiles o deciles-, se puede establecer que en Colombia la población de ingresos medios ha crecido, al tiempo que se disminuye la cantidad de personas en condiciones de pobreza (Uribe y Ramírez, 2019). Aunque desde luego existen serias críticas porque esta disminución se puede haber magnificado por cambios metodológicos en la medición (Casas y Muñoz, 2017). En relación con el sistema tributario — que debería actuar como motor de la movilidad social—, Newmant et al. (2020) ${ }^{5}$ resaltan, en la reciente demanda de inconstitucionalidad contra la integralidad de la ley del Sistema Tributario en Colombia, que este no es progresivo, siendo, además, inequitativo e injusto. Estos adjetivos indican que no se cobra más a quienes mayores ingresos tienen, porque la reforma se enfoca en impuestos indirectos (no progresividad). Además, aunque nominalmente hay progresión en las tasaciones de la renta, no se corresponden con las tasas efectivas (no equidad). Es injusta porque algunos gremios o productores - por su lobby político o por estar en zonas francas - pagan tasas menores o nulas de ciertos impuestos.

Igualmente, pese a que en apariencia el gobierno colombiano se presenta como liberal, ortodoxo, responsable técnico en el manejo de las finanzas públicas y con objetivos sociales, en la práctica ha implementado reformas que no consiguen aumentar las tasas de empleo, especialmente el formal, y que tampoco logran mejorar las finanzas del Estado (Reina, 2020). Esto también tiene que ver con la dependencia de los recursos naturales, pues cuando los precios de los commodities bajan, lo hacen también las regalías que el gobierno recibe (Alejo, Devia y Reina 2018), lo que obliga a apresurar modificaciones tributarias improvisadas (Reina, 2019).

Sin duda, hasta el 2020, antes del programa de devolución de IVA, no existían programas sociales focalizados en favor del grupo social de bajos ingresos por parte del sistema tributario. Se espera que tras su acelerada implementación en la coyuntura de la pandemia del COVID-19, este programa promueva algo de movilidad social o, al menos, mitigue en algo la pobreza extrema. En lo que respecta al sistema educativo, se han

${ }^{5}$ El 26 de Mayo de 2020 se presentó la "acción pública de inconstitucionalidad contra la integralidad del Decreto 624 de 1989, por el cual se expide el Estatuto Tributario, junto con las leyes y decretos con fuerza de ley que lo hayan modificado" por parte de los renombrados investigadores y profesores Vivian Newman Pont, Rodrigo Uprimny Yepes, Luis Jorge Garay Salamanca, Jorge Enrique Espitia Zamora, Clara Leonor Ramírez Gómez, Leopoldo Fergusson Talero, María Margarita Zuleta González, Salomón Kalmanovitz Krauter, Víctor Javier Saavedra Mercado, María Fernanda Valdés Valencia, Amaranto de Jesús Daniels Puello, Carlos Julio Salgado Araméndez, Consuelo Corredor Martínez, Jorge Iván González Borrero, Juan Camilo Cárdenas Campo, Valentina Rozo Ángel, Fernando Antonio del Niño Jesús Barberi Gómez, Astrid Martínez Ortiz, Catalina Botero Marino, Mauricio García Villegas, Esteban Hoyos Ceballos, Andrés Abel Rodríguez Villabona, Magdalena Correa Henao, Maryluz Barragán González, Mauricio Albarracín Caballero, Alejandro Rodríguez Llach y Alejandro Jiménez Ospina. 
tenido avances positivos, particularmente en la educación superior. Entre 2001 y 2012, las matrículas de formación técnica y tecnológica aumentaron en un 359,8\% y 340,6\%, respectivamente, mientras que, para las carreras profesionales, en el mismo periodo, el crecimiento fue solo del 59,5\% (Jaramillo, 2015). Con esto, se llegó a una tasa bruta de cobertura superior al 40\%, para inicios de la segunda década de este siglo en Colombia. Este es, sin embargo, un nivel aún bajo en relación con los países desarrollados, en los que la cobertura supera el $80 \%$.

Desafortunadamente, el Estado colombiano ha optado por mantener vigente la Ley 30 de 1992 que, en la práctica, ha desfinanciado a las universidades de carácter estatal, afectando la oferta de nuevos cupos en estos centros educativos. Ante este hecho, se entiende que la oferta ha crecido por los avances del SENA en educación técnica y tecnológica y por el crecimiento y/o aparición de nuevas universidades privadas. Se debe reconocer, sin embargo, que el cambio de la política pública "Ser Pilo Paga" por la de "Generación -E" y los incentivos de "Jóvenes en acción" constituyen programas sociales focalizados con una importante esperanza para la movilidad intergeneracional, medida por educación e incluso por ingresos.

Finalmente, en lo referente a los programas sociales —motivación inicial de este articulo- se evidencia una gran variedad de ellos con los mismos problemas de corrupción que se observan a lo largo del aparato público colombiano. Pese a este contexto negativo, no se debe desestimar su contribución en el bienestar social. Se destacan, por ejemplo, el "Programa de Alimentación Escolar" —PAE—, el suministro de kits de utensilios de aseo y cocina, así como alimentos, para desplazados o afectados por desastres naturales, y la entrega de mercados durante la cuarentena. Otro interesante programa social es el de Familias en Acción, un programa de transferencias condicionadas de inicios del presente siglo que pretende transformar los recursos en capacidades individuales de modo que les permita en ejercicio de su libertad el desarrollo, mejores niveles de vida (Ayola-Betancout, 2018)

Se deben mencionar, además, las prestaciones continuadas de servicios mediante la afiliación a Entidades Prestadoras de Salud —EPS - en calidad de subsidiados, y los programas de transferencia condicionadas de efectivo como "Familias en acción", "Jóvenes en Acción" y "Colombia Mayor", este último para apoyar a ciudadanos en su vejez. En síntesis, existe un amplio portafolio de programas sociales, de los cuales aquí se consideraron solo aquellos que operan a nivel nacional. Se considera que estos contribuyen, al menos, en mitigar la pobreza y facilitar la movilidad social. Tal vez ello explica que en los resultados del Latinbarómetro ${ }^{6}$ muchos colombianos comiencen a considerarse de clase media, como Uribe y Ramírez (2019) lo señalaron. Además, la reciente depuración del SISBEN, con la versión 4, contribuirá a realizar un gasto efectivo en población objetivo.

\section{CONCLUSIÓN}

El gasto social en Colombia tradicionalmente no tenía procesos rigurosos de focalización en sus programas sociales. Esto cambió cuando se implementó el Sistema de Identificación de Potenciales Beneficiarios de Programas Sociales (SISBEN) en 2007. Este

${ }^{6}$ Corporación Latinbarometro realiza periódicamente encuestas de opinión para los países de américa latina y disponibilizando su base de datos. Puede consultarlos en: http://www.latinobarometro.org/lat.jsp 
hecho permitió una inclusión, especialmente en temas de salud subsidiada, así como la implementación de programas sociales legitimados por este registro. Aunque sin duda habrá contribuido a la movilidad de algunos hogares en condición de pobreza inicial, las deficiencias de las metodologías de las tres primeras versiones del SISBEN ocasionaron un sobrerregistro, por lo que se puede inferir que fue baja la efectividad de la focalización realizada hasta 2019.

En materia de educación y tributación, se han implementado programas focalizados recientemente, lo cual se aplaude. Sin embargo, no se podrá decir mucho sobre ellos prontamente. Adicionalmente, se recomienda pensar en metodologías globalizadoras que superen los problemas de corrupción para realizar una aproximación empírica a la respuesta planteada en el presente articulo.

\section{REFERENCIAS}

Aguado, J. (2015). Efecto renta y efecto sustitución. En Guía para comprender la microeconomía (pp. 69-76). Madrid, España: Larousse—Ediciones Pirámide.

Alejo, D., Devia, N., y Reina, L.E. (2018). Análisis regional de Colombia y su maldición de recursos naturales: cambios institucionales tardíos. EURE, 44(131), 125-150. Recuperado de https://scielo.conicyt.cl/scielo.php?script=sci arttext\&pid=S0250-71612018000100125

Alzate, S. A. (2011). Las políticas públicas en Colombia. Insuficiencias y desafíos. Revista Forum, 1(1), 95-111. Recuperado

\section{http://revistas.unal.edu.co/index.php/forum/article/viewFile/32359/32374}

Angulo, R., Azevedo, J, Gaviria, A., y Páez, G. (2014). Movilidad social en Colombia. En A. Montenegro y M. Meléndez (Comp.), Equidad y Movilidad Social: diagnósticos y propuestas para la transformación de la sociedad colombiana (pp. 37-67). Bogotá, Colombia: Universidad de los Andes-DNP.

Asri, V. (2019). Targeting of social transfers: Are India's poor older people left behind. World Development, 115, 46-63. Recuperado de https://www.sciencedirect.com/science/article/abs/pii/S0305750X18303991

Ayala, J. (2017). Movilidad social. En L. Galvis (Ed.), Estudios sociales del Pacífico colombiano (pp. 103-137). Bogotá, Colombia: Banco de la República de Colombia

Ayola-Betancourt, V. (2018). Condicionar, focalizar e Invertir en capital humano: El programa "Familias en Acción" o la receta internacional de lucha contra la pobreza para Colombia. Panorama Económico, 26(1), 37-58. Recuperado de https://doi.org/10.32997/2463-0470-vol.26num.1-2018-2228

Casas, J. A. y Muñoz, M. (2017). Propuesta alternativa para las líneas de indigencia y de pobreza existentes en Colombia. Cuadernos de Economía, 36(72), 179-205. Recuperado de http://dx.doi.org/10.15446/cuad.econ.v36n72.65875

Clavijo, S., Vera, N., Cuéllar, E., \& Joya, J. S. (2019). Lo "esencial" de la Reforma Pensional en Colombia. Actualidad Económica-ANIF, 1-7. Recuperado de https://www.anif.com.co/sites/default/files/investigaciones/carta-anif-pensiones0619.pdf

Congreso de Colombia. (21 de diciembre de 2001). Por la cual se dictan normas orgánicas en materia de recursos y competencias de conformidad con los artículos 151, 288, 356 y 357 (Acto Legislativo 01 de 2001) de la Constitución Política y se dictan otras disposiciones para organizar la prestación de los servicios de educación y salud, entre otros. [Ley 715 de 2001]. 
Congreso de Colombia. (27 de diciembre de 2007). Por la cual se desarrollan los artículos 356 y 357 de la Constitución Política y se dictan otras disposiciones. [Ley 1176 de 2007]. DO: 46.854.

Cortés, F. (2014). La medición multidimensional de la pobreza en México. En Boltvinik, J. et al., Multidimensionalidad de la pobreza: propuestas para su definición y evaluación América Latina y el Caribe (pp. 99-126). Buenos Aires, Argentina: CLACSO.

Cuadro-Guzmán, E., \& Jiménez-Martínez, A. (2016). Política monetaria y equidad en Colombia (1980-2010). Panorama Económico, 24, 143 - 162. Recuperado de https://doi.org/10.32997/24630470-vol.24-num.0-2016-1554

Cummins, R. (2019). Jeremy Bentham, Utility, and the Golden Triangle of Happiness. In Wealth (s) and Subjective Well-Being (pp. 67-83). Cham, Alemania: Springer.

De Hoyos, R., Attanasio, O., y Meghir, C. (2019). Targeting High School Scholarships to the Poor: The Impact of a Program in Mexico (No. w26023). Cambridge, Estados Unidos: National Bureau of Economic Research.

Departamento Nacional de Planeación. (2016). Documento CONPES 3877 de 2016. Bogotá: DNP. Recuperado de https://colaboracion.dnp.gov.co/CDT/Conpes/Econ\%C3\%B3micos/3877.pdf

Departamento Nacional de Planeación y Presidencia de Colombia. (2019). Plan Nacional de Desarrollo 2018-2022 [Documento en sitio Web]. Recuperado de https://colaboracion.dnp.gov.co/CDT/Prensa/Resumen-PND2018-2022-final.pdf

Devereux, S. (2016). Is targeting ethical?. Global Social Policy, 16(2), 166-181. Recuperado de https://journals.sagepub.com/doi/abs/10.1177/1468018116643849?journalCode=gspa

Ferrer, E. (2020). La exigibilidad directa del derecho a la salud y la obligación de progresividad y no regresividad. En M. Morales, L. Ronconi y L. Clérico (Coord.) Interamericanización de los DESCA: El caso Cuscul Pivaral de la Corte IDH (pp. 425-455). Ciudad de México, México: Instituto Max Planck de Derecho Público Comparado y Derecho Internacional Público-Instituto de Estudios Constitucionales del Estado de Querétaro—Instituto de Investigaciones Jurídicas Universidad Nacional Autónoma de México.

Filgueira, F. (2014). Hacia un modelo de protección social universal en América Latina. Santiago de Chile, Chile: CEPAL.

Fonseca, A. (2008) Los Sistemas de protección social en América Latina: Focalización vs. Universalidad (Working Paper \#4). Santiago de Chile, Chile: FAO.

Franco, I., y Reina, L. (2018). Emergencia de la Solidaridad Interterritorial en Colombia y sus nexos con el desarrollo regional, territorial y local. Desbordes, 9(1), 59-94. Recuperado de https://hemeroteca.unad.edu.co/index.php/desbordes/article/view/3218

Garzón, J. (2018). Revisión bibliográfica sobre las Transferencias Monetarias Condicionadas en Colombia. Bogotá, Colombia: Universidad Católica.

Gaviria, A. (2010). Cambio social en Colombia durante la segunda mitad del siglo XX, Documentos CEDE No. 007714. Bogotá, Colombia: Universidad de los Andes-CEDE.

González, J. (2000). Política social e indicadores sociales en Colombia: Una evaluación. Investigación \& Desarrollo, 8(3), 244-257. Recuperado de http://manglar.uninorte.edu.co/handle/10584/4415?show=full

Herrera-Jiménez, A., \& Villegas-Quino, H. (2016). Circunstancias y determinantes individuales en Bolivia: Características de los hogares e igualdad de oportunidades (2003-2013). Panorama Económico, 24, 17 - 48. 
Hernández, D., Orozco, C., y Vázquez, B. (2005). La focalización como estrategia de política pública, Serie: documentos de investigación No. 25. México: Secretaría de Desarrollo Social.

Huber, E. (2002). Models of Capitalism: Lessons for Latin América. Pennsylvania: Pennsylvania State University Press.

Huber, E., y Stephens, J. (2004). Combating Old and New Social Risks. En 14th International Conference of Europeanists, Palmer House Hilton.

Ibarra, F. (2019). Análisis de la estrategia de focalización para otorgar el subsidio de aseguramiento en salud: el caso Medellín 2018. Universidad de Antioquia, Medellín, Colombia.

Jaramillo, J. A. V. (2015). Educación superior en Colombia: ¿crisis o realidad? Revista Divergencia (Artículos Sobre Economía), (18), 14-20. Recuperado de https://revistas.uexternado.edu.co/index.php/diver/article/view/4170/4616

Llanes-Dueñas, L. (2017). Los efectos de la dinámica social sobre el crecimiento económico: aplicación al departamento del Meta (Colombia). Panorama Económico, 25(3), 423-442. Recuperado de https://doi.org/10.32997/2463-0470-vol.25-num.3-2017-2084

Max-Neef, M., Elizalde, A., y Hopenhayn, M. (2006). Desarrollo a escala humana: conceptos, aplicaciones y algunas reflexiones. Barcelona, España: Icaria Editorial.

Nechyba, T. J. (2000). Mobility, targeting, and private-school vouchers. American Economic Review, 90(1), 130-146. Recuperado de https://www.jstor.org/stable/117284? seq=1

Newman, V.; et al. (26 de mayo de 2020). Acción pública de inconstitucionalidad contra la integralidad del Decreto 624 de 1989, por el cual se expide el Estatuto Tributario, junto con las leyes y decretos con fuerza de ley que lo hayan modificado [Documento en sitio Web]. Recuperado de https://www.dejusticia.org/wp-content/uploads/2020/05/Demanda-EstatutoTributario-Dejusticia-2020.pdf

Ocampo, J. A. (2008). Las concepciones de la política social: Universalismo versus focalización. Nueva Sociedad, 215, 36-61.

Pautassi, L (2020) La agenda de progresividad en el Sistema Interamericano: una aproximación a partir del derecho a la salud. En M. Morales, L. Ronconi y L. Clérico (Coord.) Interamericanización de los DESCA: El caso Cuscul Pivaral de la Corte IDH (pp. 761-800). Ciudad de México, México: Instituto Max Planck de Derecho Público Comparado y Derecho Internacional Público_Instituto de Estudios Constitucionales del Estado de Querétaro-Instituto de Investigaciones Jurídicas Universidad Nacional Autónoma de México.

Raczynski, D. (1995). Focalización de programas sociales: lecciones de la experiencia chilena. En D. Raczynski y C. Pizarro, Políticas económicas y sociales en el Chile democrático (pp. 217-224). Santiago de Chile: Unicef. Recuperado de http://www.cieplan.cl/media/publicaciones/archivos/15/Capitulo 10.pdf

Reina, L. (2017a). Economía del Bienestar [Vídeo]. Bogotá: UNAD. Recuperado de http://hdl.handle.net/10596/11640

Reina, L. (2017b). Políticas Públicas e Indicadores Sociales [Vídeo]. Bogotá: UNAD. Recuperado de http://hdl.handle.net/10596/12634

Reina, L. (2018). Lo alternativo en los estudios y prácticas del desarrollo. Documentos de Trabajo ECSAH, 2018 (2), 1-12. Recuperado de https://doi.org/10.22490/ECSAH.2590

Reina, L. (2019). Efecto de volatilidad del precio del petróleo en la economía global. En Evento «Filosofía en Red». UNAD, Bogotá, Colombia. 
Reina, L. (2020). El papel social y fiscal de la disminución de impuestos a grandes empresas. En: Bogliacino (Ed./Comp.) \#21N (Investigaciones y Productos No. 33) (pp. 86-90). Bogotá, Colombia: Centro de Investigaciones para el Desarrollo - CID.

Revéiz, É. (2016). La transgresión moral de las élites y el sometimiento de los Estados: cooptación o democracia. Bogotá, Colombia: Academia Colombiana de Ciencias Económicas.

Rivera-Sotelo, A. (2011). El utilitarismo de Jeremy Bentham ¿fundamento de la teoría de Leon Walras? Cuadernos de Economía, 30(55), 55-76. Recuperado de http://www.scielo.org.co/scielo.php?script=sci arttext\&pid=S0121-47722011000200003

Roberts, B. (14 y 15 de diciembre de 2001). Las nuevas políticas sociales en América Latina y el desarrollo de la ciudadanía: una perspectiva de interfaz. En Taller Agencia, Conocimiento y poder: Nuevas Direcciones. Universidad de Wageningen, Wageningen, Países Bajos.

Ruiz, F., Botello, P., y Marín, N. (2014). Convergencia espacial de la Pobreza Multidimensional local con enfoque diferencial en América Latina. En Boltvinik, J. et al., Multidimensionalidad de la pobreza: propuestas para su definición y evaluación América Latina y el Caribe (pp. 187-232). Buenos Aires: CLACSO.

Salgado-Hernández, Y., Martínez-Dorantes, O., \& Meireles, M. (2018). Programas de transferencias monetarias condicionadas, pobreza y desarrollo: un análisis comparado de Bolsa Familia (Brasil) y Prospera (México). Panorama Económico, 26(1), 13-36. Recuperado de https://doi.org/10.32997/2463-0470-vol.26-num.1-2018-2229

Sánchez, P (2018). La política pública como garantía de derechos de la primera infancia en Colombia. Derecho Y Realidad, 16(31), 1-16. Recuperado de https://doi.org/10.19053/16923936.v16.n31.2018.9105

Sen, A. (1999) Desarrollo y libertad. Buenos Aires: Planeta.

Sen, A. (2003). La economía política de la focalización. Comercio Exterior, 53(6), 555-562. Recuperado de http://revistas.bancomext.gob.mx/rce/magazines/53/7/RCE.pdf

Senhoras, E. (2003). Políticas públicas nos anos noventa: um ensaio sobre as causas e implicações da crise do Estado brasileiro de Bem Estar Social. Revista Oikos, 2(2).

Senhoras, E. (2015). Polêmicas sobre a legislação federal de cotas Étnico-raciais no Brasil. Revista Em Tempo, v. 14.

Stiglitz, J. E. (2003). La economía del sector público. Barcelona: Antoni Bosch.

Subramanian, S. (2019). Targeting Assistance to the Poor. En Inequality and Poverty: a short critical introduction (pp. 65-67). Singapur: Springer.

Torres-Melo, J., \& Santander, J. (2013). Introducción a las políticas públicas: conceptos y herramientas desde la relación entre Estado y ciudadanía. IEMP Ediciones.

Uribe, C. y Ramírez, J. (2019). Clase media y movilidad social en Colombia. Revista Colombiana de Sociología, 42(2), 229-255. Recuperado de https://doi.org/10.15446/rcs.v42n2.50749

Viáfara, C. (2017). Movilidad social intergeneracional de acuerdo al color de la piel en Colombia. En Sociedad y economía, (33), 263-287. Recuperado de http://www.scielo.org.co/scielo.php?script=sci abstract\&pid=\$1657-63572017000200263

Zapata Muñoz, K. V., \& Valencia Salazar, S. (2018). Acceso a la salud de personas en condición de discapacidad del régimen subsidiado, en los años 2015 y 2016 en Colombia (tesis doctoral). Fundación Universitaria del Área Andina. 